\section{(2) OPEN ACCESS}

\title{
Essential role of smooth muscle Rac1 in severe asthma-associated airway remodelling
}

\author{
Florian Dilasser, ${ }^{1}$ Lindsay Rose, ${ }^{1}$ Dorian Hassoun, ${ }^{1}$ Martin Klein, ${ }^{1}$ Morgane Rousselle, \\ Carole Brosseau, ${ }^{2}$ Christophe Guignabert, ${ }^{3}$ Camille Taillé, ${ }^{4}$ Marie Christine Dombret ${ }_{1}$ \\ Leonarda Di Candia, ${ }^{4}$ Nicolas Heddebaut, ${ }^{6}$ Gregory Bouchaud, ${ }_{1}{ }^{1}$ Marina Pretolani, ${ }_{1}^{6}$ \\ Antoine Magnan, ${ }^{7}$ Gervaise Loirand, ${ }^{1}$ Vincent Sauzeau ${ }^{1}$
}

\begin{abstract}
Inserm UMR 1087, Nantes, France

${ }^{2}$ Centre de recherche en transplantation, Inserm 1064, Nantes, France

${ }^{3}$ INSERM U999, Le Plessis Robinson, France

${ }^{4}$ Service de Pneumologie et Centre de Référence des Maladies Pulmonaires Rares, Hôpital Bichat - Claude-Bernard, Paris, France

${ }^{5}$ APHP, Paris, France

IINSERM 1152, Paris, France

${ }^{7}$ Institut du Thorax UMR1087 CNRS 6291, INSERM, Université de Nantes, CHU de Nantes, DHU2020, Nantes, France
\end{abstract}

\section{Correspondence to} Dr Vincent Sauzeau, Inserm UMR 1087, Nantes, France; vincent.sauzeau@inserm.fr

FD and LR contributed equally.

Received 28 September 2020 Revised 20 November 2020 Accepted 2 December 2020

Published Online First

4 February 2021
ABSTRACT
Background Severe asthma is a chronic lung

disease characterised by inflammation, airway

hyperresponsiveness (AHR) and airway remodelling. The

molecular mechanisms underlying uncontrolled airway

smooth muscle cell (aSMC) proliferation involved in

pulmonary remodelling are still largely unknown. Small

$G$ proteins of the Rho family (RhoA, Rac1 and (dc42)

are key regulators of smooth muscle functions and we recently demonstrated that Rac1 is activated in aSMC from allergic mice. The objective of this study was to assess the role of Rac1 in severe asthma-associated airway remodelling.

Methods and results Immunofluorescence analysis in human bronchial biopsies revealed an increased Rac1 activity in aSMC from patients with severe asthma compared with control subjects. Inhibition of Rac1 by EHT1864 showed that Rac1 signalling controlled human aSMC proliferation induced by mitogenic stimuli through the signal transducer and activator of transcription 3 (STAT3) signalling pathway. In vivo, specific deletion of Rac1 in SMC or pharmacological inhibition of Rac1 by nebulisation of NSC23766 prevented AHR and aSMC hyperplasia in a mouse model of severe asthma. Moreover, the Rac1 inhibitor prevented goblet cell hyperplasia and epithelial cell hypertrophy whereas treatment with corticosteroids had less effect. Nebulisation of NSC23766 also decreased eosinophil accumulation in the bronchoalveolar lavage of asthmatic mice.

Conclusion This study demonstrates that Rac1 is overactive in the airways of patients with severe asthma and is essential for aSMC proliferation. It also provides evidence that Rac1 is causally involved in AHR and airway remodelling. Rac1 may represent as an interesting target for treating both AHR and airway remodelling of patients with severe asthma.

\section{INTRODUCTION}

Check for updates

(C) Author(s) (or their employer(s)) 2021. Re-use permitted under CC BY-NC. No commercial re-use. See rights and permissions. Published by BMJ.

To cite: Dilasser F, Rose $L$, Hassoun $\mathrm{D}$, et al. Thorax 2021;76:326-334.
Asthma is a heterogenous and complex disease that affects more than 300 million people worldwide. ${ }^{12}$ The disease expression includes wheezy dyspnoea, expiration blocking, cough and thoracic oppression in a context of chronic bronchial inflammation. ${ }^{3}$ Current treatments are based on anti-inflammatory therapies (inhaled and oral corticosteroids) and inhaled bronchodilators. Severe asthma is defined as asthma that is not improved by standard treatment and that requires a combination of high doses of inhaled corticosteroids with an add-on therapy to

\section{key message}

What is the key question?

- The objective of this study was to assess the role of Rac1 in severe asthma-associated airway remodelling.

What is the bottom line?

- The current study unveils an overactivation of smooth muscle Rac1 in bronchi from severe asthmatics, and highlights a leading role of Rac1 in airway remodeling.

Why read on?

- Rac1 appears as a new attractive therapeutic target in severe asthma.

be controlled, or that remains uncontrolled or even worsens despite these treatments. ${ }^{4}$ Severe asthma leads to a poor quality of life and important healthcare expenses due to direct (care visits and treatments) and indirect (day off work) costs. ${ }^{5}$ Thus, improving the therapeutic management of these patients represents a major public health challenge.

In addition to chronic inflammation, severe asthma is characterised by airway hyperresponsiveness (AHR) and structural changes of the airway wall. This airway remodelling includes goblet cell hyperplasia, thickening of the basal membrane, angiogenesis and airway smooth muscle cells (aSMCs) hypertrophy and hyperplasia. ${ }^{7-9}$ The extent of this remodelling correlates with the severity of asthma and the degree of airflow obstruction. ${ }^{10}$ Currently, the only available treatment targeting airway remodelling is bronchial thermoplasty, a bronchoscopy procedure that consists in reducing the aSMC mass through the local deliver of controlled radiofrequency energy. Although bronchial thermoplasty has been shown to be effective in controlling asthma in severe asthmatics, ${ }^{11-13}$ its long-term effects are not known and the selection of patients who could benefit from this invasive procedure remains challenging. Nevertheless, the use of this procedure demonstrated the therapeutic value of targeting aSMCs in severe asthma and the need of developing new pharmacologic strategies for limiting aSMC proliferation and airway remodelling in severely affected patients.

It has been widely demonstrated in vitro that the small $G$ protein Rac1 activity controls aSMC 
proliferation. ${ }^{14} 15$ We thus hypothesised that Rac1 may be implicated in the proliferation of aSMC and consequently in the deleterious airway remodelling associated with severe asthma. By using human bronchial biopsies, human aSMC cultures and a mice model of severe allergic asthma sensitised to house dust mite (HDM) that closely mimics human pathology, we demonstrated that activation of Rac1 is causally involved in aSMCs proliferation and airway remodelling associated with severe asthma. We also show that in vivo pharmacological inhibition of Rac1 prevents asthma-associated airway remodelling thus confirming Rac1 as an alternative potential target for the treatment of severe asthma.

\section{METHODS}

\section{Human bronchial biopsies}

Bronchial biopsies were obtained by bronchial endoscopy from severe asthmatics as previously described. ${ }^{16}$ All enrolled patients gave written approval. Control samples were obtained from donor lung transplants under $\mathrm{NaRacAS}$ (expression and activity of Rac1 in bronchial smooth muscle cells from from asthmatic patients) protocol (NCT03325088). Clinical protocols were previously approved by relevant ethic committees.

\section{Analysis of Rac1 activity}

Human pulmonary biopsies paraffin-embedded sections were deparaffinised and permeabilised (phosphate buffered saline $(\mathrm{PBS})+0.1 \%$ Triton-X100) before incubation with anti-Rac-GTP antibody (26903, NewEast Biosciences, King of Prussia, Pennsylvania) (1/1000) overnight at room temperature (RT). After three washes in PBS, sections were incubated for 1 hour at RT with the secondary Alexa568-labelled anti-rabbit antibody (1/1000). AntiSM22 $\alpha$ antibody (Abcam) $(1 / 500 \mathrm{O} / \mathrm{N}$ at RT) with Alexa488labelled anti-mouse antibody (1/1000 1 hour at RT) were used to localise smooth muscle. To quantify Rac-GTP levels within the smooth muscle, Rac-GTP fluorescence intensities were measured inside a mask delimited by SM22a-positive cells and normalised to the control condition.

\section{aSMCs proliferation}

Primary aSMCs were isolated from human bronchial biopsies. Additional detail on the method is provided in an online data supplement. Human aSMCs were seeded into 24-well plates (10 000 aSMCs/well) and allowed to adhere during 6 hour before serum starvation during 24 hours. When indicated, human aSMCs were treated with the Rac inhibitor, EHT1864 $\left(10^{-5} \mathrm{M}\right.$; Tocris Bioscience), P21-activated kinases (Pak) inhibitor IPA3 $\left(10^{-5} \mathrm{M}\right.$; Tocris Bioscience), Akt inhibitor VIII (10 ${ }^{-5} \mathrm{M}$; Calbiochem), MEK inhibitor PD98059 (10 ${ }^{-5} \mathrm{M}$; ThermoFisher), JAK inhibitor ruxolitinib $\left(10^{-5} \mathrm{M}\right.$; InvivoGen) added $30 \mathrm{~min}$ before stimulation with bFGF (25 ng/mL; Miltenyi Biotech), PDGF-bb (25 ng/mL; Miltenyi Biotech), interleukins (IL)-13 (10 ng/mL; Miltenyi Biotech), IL-33 (10 ng/mL; Miltenyi Biotech), IL-17 (20 ng/mL; Miltenyi Biotech), IL-9 (10 ng/mL; Miltenyi Biotech) or TSLP (10 ng/mL; Miltenyi Biotech) for 48 hours. Cells were stained with EdU for 12 hours at $10^{-5} \mathrm{M}$ according to the manufacturer's indications (EdU Staining Proliferation Kit iFluor 488, ID: ab219801, Abcam). Proliferation was quantified by the ratio of EdU-positive cells over the total number of cells. Proliferative signalling pathways were analysed by immunoblotting detailed in an online data supplement.

\section{Allergic asthma models}

$\mathrm{C} 57 \mathrm{Bl} / 6 \mathrm{Rac} 1^{\text {lox/lox }}$ and SMMHC-Rac1 ${ }^{\text {lox/lox }}$ mice were obtained as previously described. ${ }^{17}$ Rac1 deletion in smooth muscle cells (SMCs) was induced in 8-week-old SMMHC-Rac1 $1^{\text {lox/lox }}$ males by intraperitoneal injection of tamoxifen $(1 \mathrm{mg} /$ day in sunflower oil) for five consecutive days during 2 weeks. Tamoxifen-treated $\mathrm{Rac} 1^{\text {lox/lox }}$ mice were used as control. Allergic asthma was induced in mice using a total HDM extract (Dermatophagoides farinae, from Stallergenes Greer, Antony, France), as described previously. ${ }^{18}$ Additional details on the method for experimental models and the analyses of bronchoalveolar lavage fluid and airways reactivity are provided in an online data supplement.

\section{Histology}

To assess smooth muscle hypertrophy/hyperplasia, lung sections were stained by immunochemistry with SM22a antibody (Abcam). Hyperplasia was expressed as the ratio of SM22a-positive area to the total bronchial area and normalised to the control condition. Additional detail on the method for making pulmonary sections is provided in an online data supplement.

\section{Statistics}

Mann-Whitney test was performed for two-group comparisons. For multiple comparisons, the non-parametric Kruskal-Wallis test was used followed by Dunns' post-test to specifically compare indicated groups. The two-way ANOVA test was used for multiple comparisons of bronchial contractility studies. Data analysis was performed using the GraphPad Prism software. The threshold for statistical significance was set at $\mathrm{p}<0.05$.

\section{RESULTS \\ Rac1 activity is increased in aSMC from patients with severe asthma}

To validate the potential role of Rac1 in airway remodelling associated with severe asthma, we determined the level of Rac1 activity in bronchial biopsies from healthy subjects and from patients with severe asthma (table 1). Asthmatic donors of bronchial biopsies were mainly 50-year-old female patients with adult asthma onset. They suffered from hypereosinophilic exacerbation-prone asthma for a mean duration of 24.7 years, with pulmonary function tests moderately altered but predominantly characterised by fixed airflow obstruction. These patients were uncontrolled (mean values of asthma control test $=8.5$ ) and they showed poor quality of life (mean values of Asthma Quality-of-Life Questionnaire=2.3) (table 1), despite an optimal treatment with high doses of inhaled corticosteroids associated with another controller, sometimes in combination with oral corticosteroids. Immunofluorescence measurements of Rac1 activity, using an antibody that specifically recognises the active GTP-bound form of Rac1, revealed a greater intensity in bronchial biopsies from asthmatic patients than from controls (figure 1A,B). Labelling of aSMC by anti-SM22a antibody showed that the aSMC area was larger in bronchial biopsies of severe asthmatics than in control subjects (figure 1A,B). Furthermore, the high level of Rac1-GTP detected in biopsies of patients with severe asthma is mainly localised in SM22a-positive area indicating that it results from an increase in Rac1 activity in aSMC (figure 1A,B). The activation of Rac1 observed within the aSMC is thus consistent with our hypothesis of a potential role of Rac1 signalling in airway remodelling and in aSMC hyperplasia in severe asthmatics.

\section{aSMCs proliferation depends on Rac1 activity}

Several growth factors, cytokines and chemokines have been proposed to participate in airway remodelling in severe asthma by promoting aSMC proliferation. ${ }^{19-24}$ We therefore assessed the role of Rac1 in primary human aSMC proliferation in response to different mitogenic stimuli. Among the mitogen factors used, only bFGF and PDGFbb induced a significant proliferation of control 


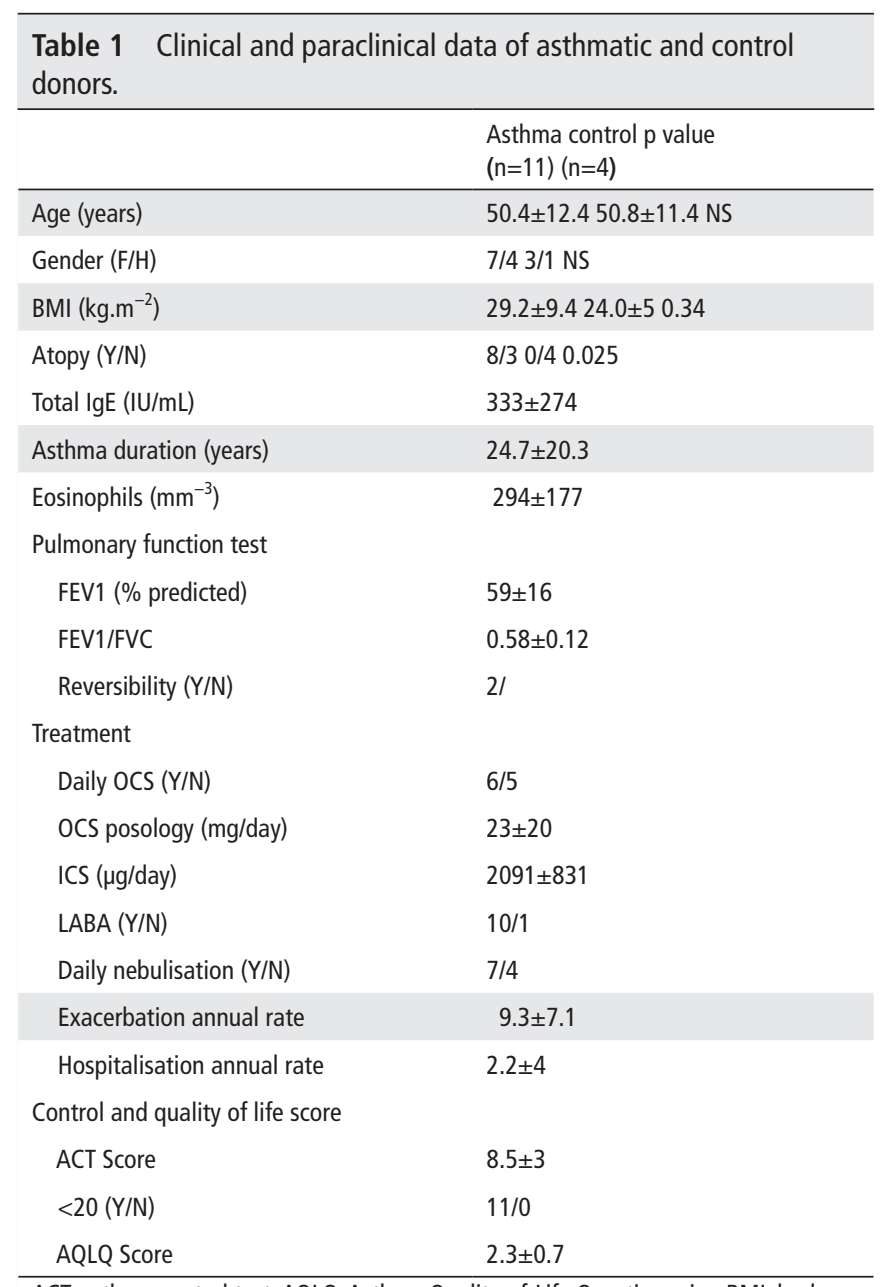

ACT, asthma control test; AQLQ, Asthma Quality-of-Life Questionnaire; BMI, body mass index; FEV1, Forced Expiratory Volume in one second; FVC, forced vital capacity; ICS, inhaled cortico steroids; IgE, immunoglobulin E; LABA, long acting beta2 agonists; OCS, oral cortico steroids.SEM). Fisher statistical test was used to compare Control and Asthmatic patients.

human aSMC (online supplemental figure E1). Interestingly, both in basal condition and on exposure to bFGF, the proliferation rate of aSMC from severe asthma patients was significantly higher than that of aSMC from control subjects (figure 2A,B). These differences are abolished by the Rac inhibitor, EHT1864, that prevented both spontaneous and bFGF- and PDGFbb-induced proliferation of aSMC from control and severe asthmatics (figure $2 \mathrm{~A}, \mathrm{C}$ ). These results suggest that Rac1 activity is involved in the mitogenic effect of bFGF and PDGFbb on human aSMC and participates to the high proliferation rate of aSMC of asthmatic patients. To validate this hypothesis, we assessed the activation of the Rac1 signalling pathway, by measuring the phosphorylation of Pak, one of the main downstream targets of Rac1, by western blot (figure 3A). Stimulation of control aSMC with bFGF and PDGFbb indeed increased Pak phosphorylation and this response was prevented by EHT1864, thereby confirming the activation of Rac1 (figure 3A).

\section{Rac1/Pak1/STAT3 signaling pathway is involved in growth factor-induced aSMCs proliferation}

Transduction pathways activated by mitogens involved in asthmatic airway remodelling have been shown to converge to a relatively limited number of intracellular signalling modules, mainly P44/42 mitogen-activated protein kinases (MAPK), phosphoinositide 3-kinase (PI3K)/Akt and JAK/STAT. ${ }^{24-28}$ Indeed, we confirm that bFGF and PDGFbb rapidly increased the phosphorylation levels of P44/42, Akt, and signal transducer and activator of transcription 3 (STAT3) in control human aSMCs (figure 3A and online supplemental figure E2). Inhibition of these signalling pathways by PD98059, Akt VIII inhibitor and ruxolitinib, respectively, reduced the proliferation of control human aSMCs at baseline and after bFGF and PDGFbb stimulation, attesting the role of these signalling pathways in this process (figure $3 \mathrm{~B}$ ). Phosphorylation of STAT3 induced by bFGF and PDGFbb was prevented by the Rac1 inhibitor EHT1864 (figure 3A), which had no effect on P44/42 activation in response to the two mitogenic factors (online supplemental figure E2). EHT1864 also downregulated Akt phosphorylation in bFGF-treated human aSMC, but not in aSMC stimulated by PDGFbb (online supplemental figure E2). These results demonstrated the essential role of Rac1 in the activation of the STAT3 pathway by mitogenic stimuli in human aSMC.

\section{SM Rac1 deletion prevents airway remodeling in a mouse model of severe asthma}

To establish the role of Rac1 in airway remodelling in vivo, we developed a murine model of severe allergic asthma induced by a percutaneous sensitisation and repeated intranasal challenges with HDM. This model allows the observation of major changes of the airway wall including aSMCs hyperplasia (figure 4A,B), AHR of bronchial rings to methacholine (figure 4C), and mixed inflammation with eosinophil and neutrophil accumulation in bronchoalveolar lavage (BAL) fluid (figure 4D). Nebulisation of the reference corticosteroid, beclomethasone at $150 \mu \mathrm{g} / \mathrm{kg}^{29} \mathrm{had}$ no significant effect on airway inflammation in this severe asthma model, whereas it prevented BAL eosinophilia and neutrophilia in an acute allergic asthma model (online supplemental figure E3). These results show that this severe allergic asthma model closely mimics the main features of severe asthma in humans, including the corticosteroid resistance.

We next submitted tamoxifen-inducible SM-Rac1-KO mice ${ }^{14}$ to the severe allergic asthma protocol. ASM area and AHR were significantly reduced in SM-Rac1-KO mice compared with SM-Ra$\mathrm{c} 1^{\text {lox/lox }}$ (figure 4B,C), whereas the histological grade, inflammatory cells accumulation in BAL and mucus production (figure 4A,D) remained unchanged. These results suggest a causal role of Rac1 in SMC hyperplasia and the resulting airway remodelling associated with severe allergic asthma.

\section{Inhalation of a Rac1 inhibitor prevents aSMC hyperplasia, $A H R$, and pulmonary inflammation in a murine allergic severe asthma model}

As a proof of concept to demonstrate the therapeutic value of pharmacological inhibition of Rac1 to limit airway remodelling associated with severe asthma, the Rac1 inhibitor NSC23766 was administrated by repeated nebulisations before each HDM challenge. NSC23766 abrogated SMC hyperplasia and AHR of bronchial rings in response to methacholine (figure 5A-C), but also peri-bronchial/ vascular infiltrates of inflammatory cells (figure $5 \mathrm{~A}, \mathrm{~B}$ ). This effect of NSC23766 on inflammatory cell infiltration was confirmed by the significant decrease of the number of macrophages and eosinophils in BAL fluid of NSC23766-treated mice, as compared with vehicle-treated mice (figure 5D). The efficiency of NSC23766 on airway remodelling and pulmonary inflammation was shown to be higher than that of current reference treatments such as repeated high doses of beclomethasone inhalations $(1500 \mu \mathrm{g} / \mathrm{kg})$, or the longlasting $\beta 2$-agonist, formoterol (figure 5A,B,D). Despite a significant reduction of inflammatory cell infiltrate, beclomethasone failed to 
A
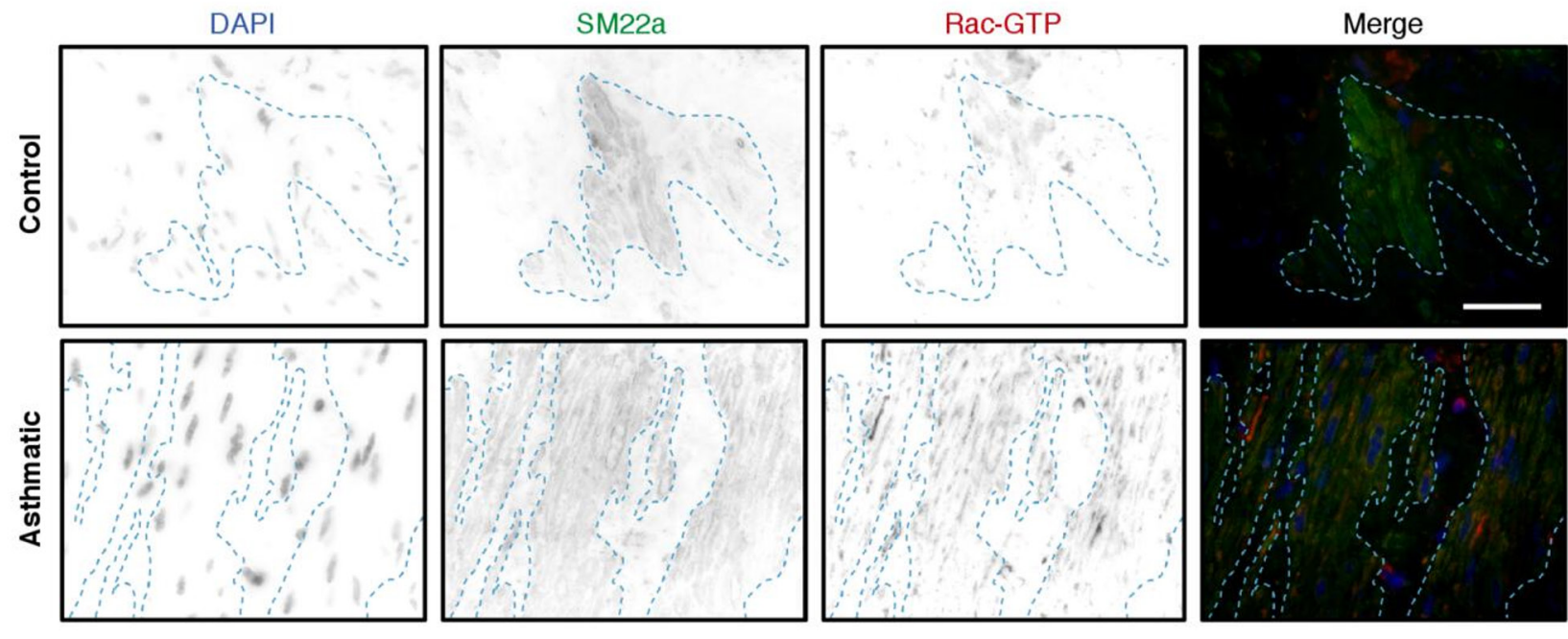

B
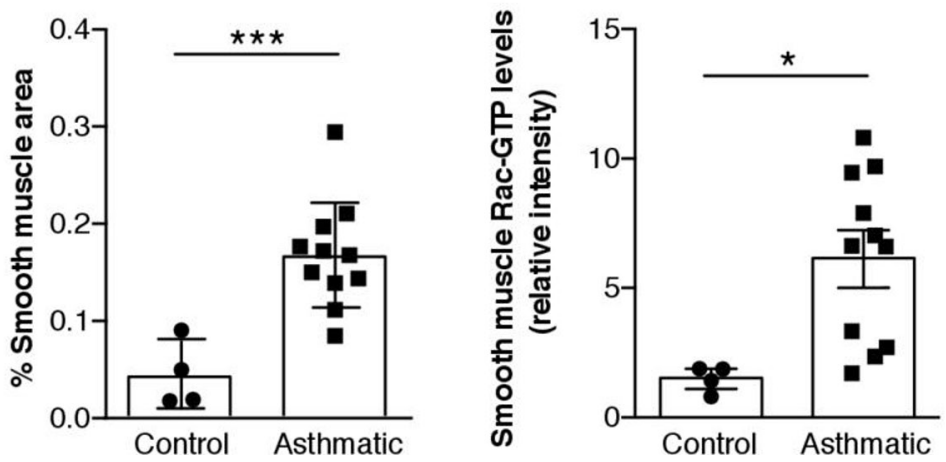

Figure 1 Rac activity is increased in airway smooth muscle contained in bronchial biopsies from asthmatics. (A) Representative images of Rac-GTP immunofluorescence $(\mathrm{red})$ in biopsies sections from control $(n=4)$ and patients with severe asthma $(n=11)$. Nuclei were detected by $4^{\prime}, 6$-diamidino-2phenylindole staining (blue) and smooth muscle by SM22a immunofluorescence (green). Scale bar, $100 \mu \mathrm{m}$. (B) Quantification of smooth muscle area, mean fluorescence intensity of the Rac1-GTP signal within the biopsy and within the smooth muscle layer. Data are presented as mean \pm SEM. MannWhitney statistical test was used to compare control and asthmatic groups. ${ }^{*} \mathrm{P}<0.05,{ }^{* * *} \mathrm{p}<0.001$.

prevent aSMC hyperplasia, and formoterol had no effect on lung inflammation and remodelling.

\section{DISCUSSION}

Our current study revealed a leading role of Rac1 in airway remodelling and in aSMC hyperplasia associated with severe asthma, by promoting STAT3-dependent aSMC proliferation. This involvement of Rac1 in the pathological remodelling in the human disease is consistent with its overactivation observed in bronchi from severe asthmatics.

The increase of aSMC mass is one of the main features of airway remodelling associated with asthma and is considered as a marker of disease severity. ${ }^{12}{ }^{19}$ It relies on a high proliferation rate of aSMC in patients with severe asthma compared with mild and moderate asthma, or control subjects. ${ }^{30}$ A number of mediators can operate in concert to stimulate aSMC proliferation, including growth factors (PDGFbb and bFGF), cytokines and chemokines, which are produced by inflammatory and airway structural cells, and by products of mast cells infiltrating the aSMC bundles, such as histamine, tryptase, and leukotrienes. ${ }^{22}$ Although initially described as the main intracellular signalling pathway mediating cytokine responses, the JAK/STAT signalling pathway has been shown to be activated by many different ligands and receptors, including growth factor/tyrosine kinase receptors and $G$ protein coupled receptors. ${ }^{31}$ Consistent with our results, Rac1 has been shown to be required for growth factor receptor-mediated and $\mathrm{G}$ protein coupled-receptor-mediated activation of the JAK/STAT pathway, thus defining Rac1 as a hub in signalling networks that control human aSMC proliferation. ${ }^{24} 3233$ This role of Rac1 is in agreement with the strong activity of Rac1 systematically observed in the remodelled airway wall of patients with severe asthma compared with control subjects, even though the number of samples analysed was limited. Recently, P-Rex1, a Rac1 exchange factor, has been shown to be aberrantly upregulated in lung tissue from patients with asthma and to potentiate growth factor-induced human aSMC proliferation. ${ }^{34}$ Therefore, it can be hypothesised that the activation of aSMC Rac1 observed in severe asthma patients could be related, at least in part, to this aberrant upregulation of P-Rex1 expression, and would be responsible for the increased aSMC proliferation in asthma.

The increase in aSMC mass in asthma patients was associated with airflow obstruction. ${ }^{9}{ }^{10}$ Indeed, aSMC are not only responsible for AHR through their contractile activity, but also contribute to the inflammatory process by modifying the extracellular matrix and producing mediators that act on inflammatory cells. Pharmacological targeting of aSMC thus appears as an attractive strategy for novel asthma therapies. Current therapeutic strategies remain based on the chronic use of high dose inhaled or oral corticosteroids, resulting in various and harmful long-term side effects. ${ }^{35} 36 \mathrm{New}$ therapeutic strategies such as monoclonal antibodies anti-IgE (omalizumab), anti-IL-5 (mepolizumab, reslizumab), anti-IL-5 receptor 
A

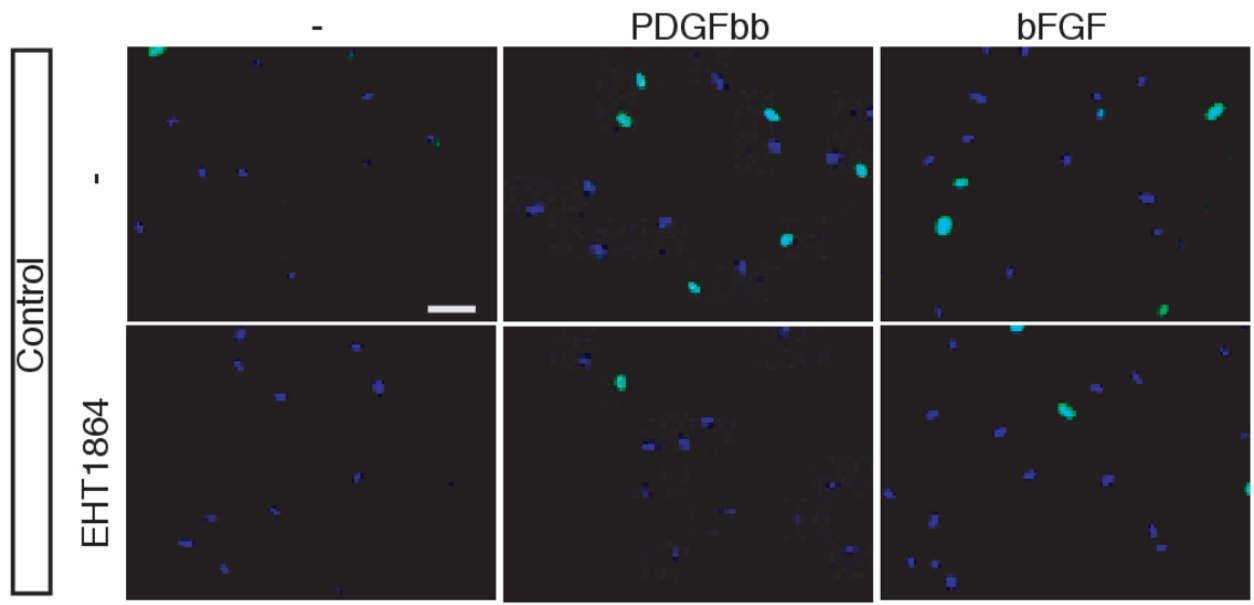

B

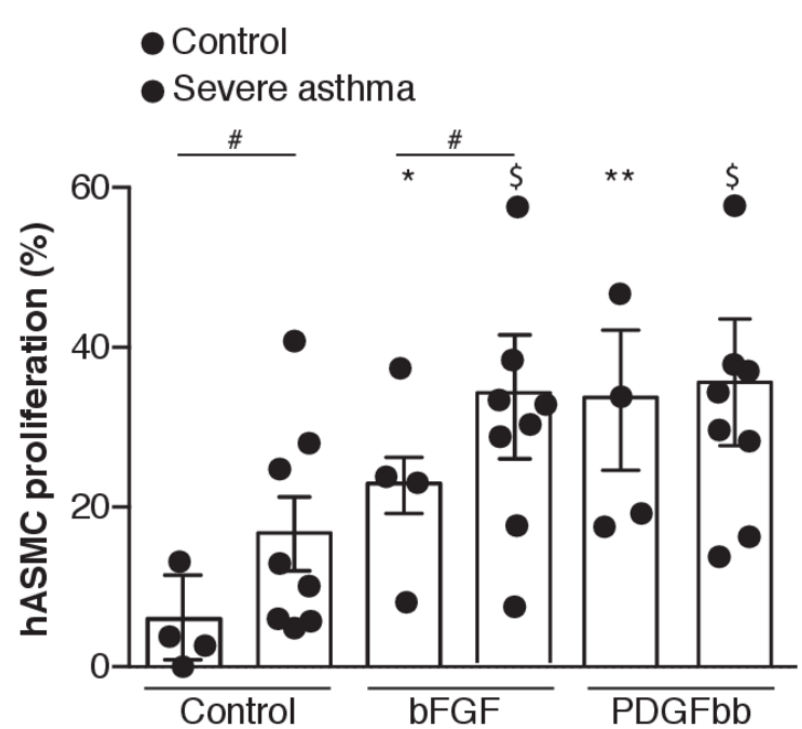

C

- Control

Severe asthma

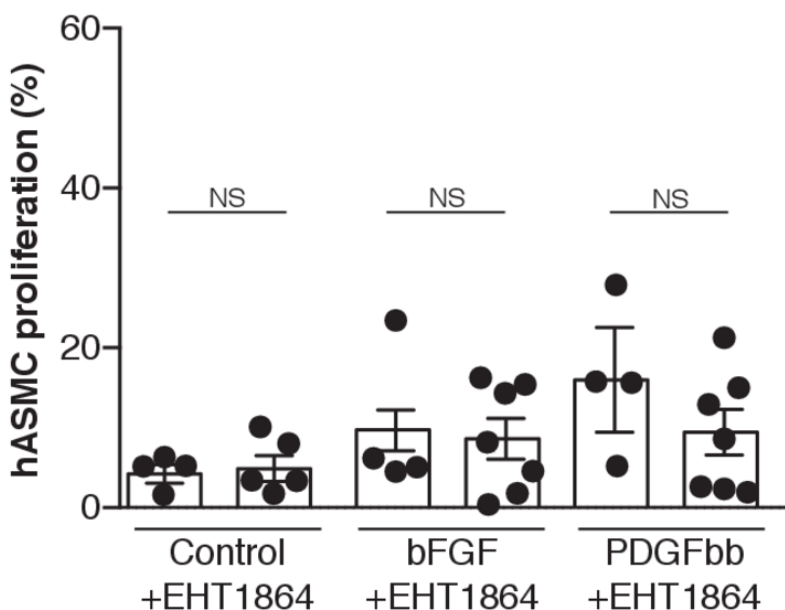

Figure 2 Rac1 inhibition reduces bFGF-induced and PDGFbb-induced aSMCs proliferation. (A) Representative images of airway smooth muscle cell (aSMC) proliferation from control and severe asthmatics induced by bFGF and PDGFbb, in the absence and in the presence of the Rac1 inhibitor, EHT1864. Nuclei are detected by 4',6-diamidino-2-phenylindole staining (blue) and aSMC proliferation by EdU staining (green). Scale bar, $25 \mu \mathrm{m}$. $(B, C)$ Quantification of aSMC proliferation by EdU staining in the absence (B) and in the presence (C) of EHT1864. The results are expressed as the percentage of cell proliferation (EdU-positive cells) (mean \pm SEM of $n=3$ independent experiments). Kruskal-Wallis test followed by Dunns' posttest were used. ${ }^{*} P<0.05,{ }^{*} \mathrm{P}<0.01$ versus untreated cells from control subjects; ${ }^{\$} \mathrm{P}<0.05$ versus untreated cells from patients with severe asthma; $\# \mathrm{p}<0.05$ versus cells from control subjects. 
A
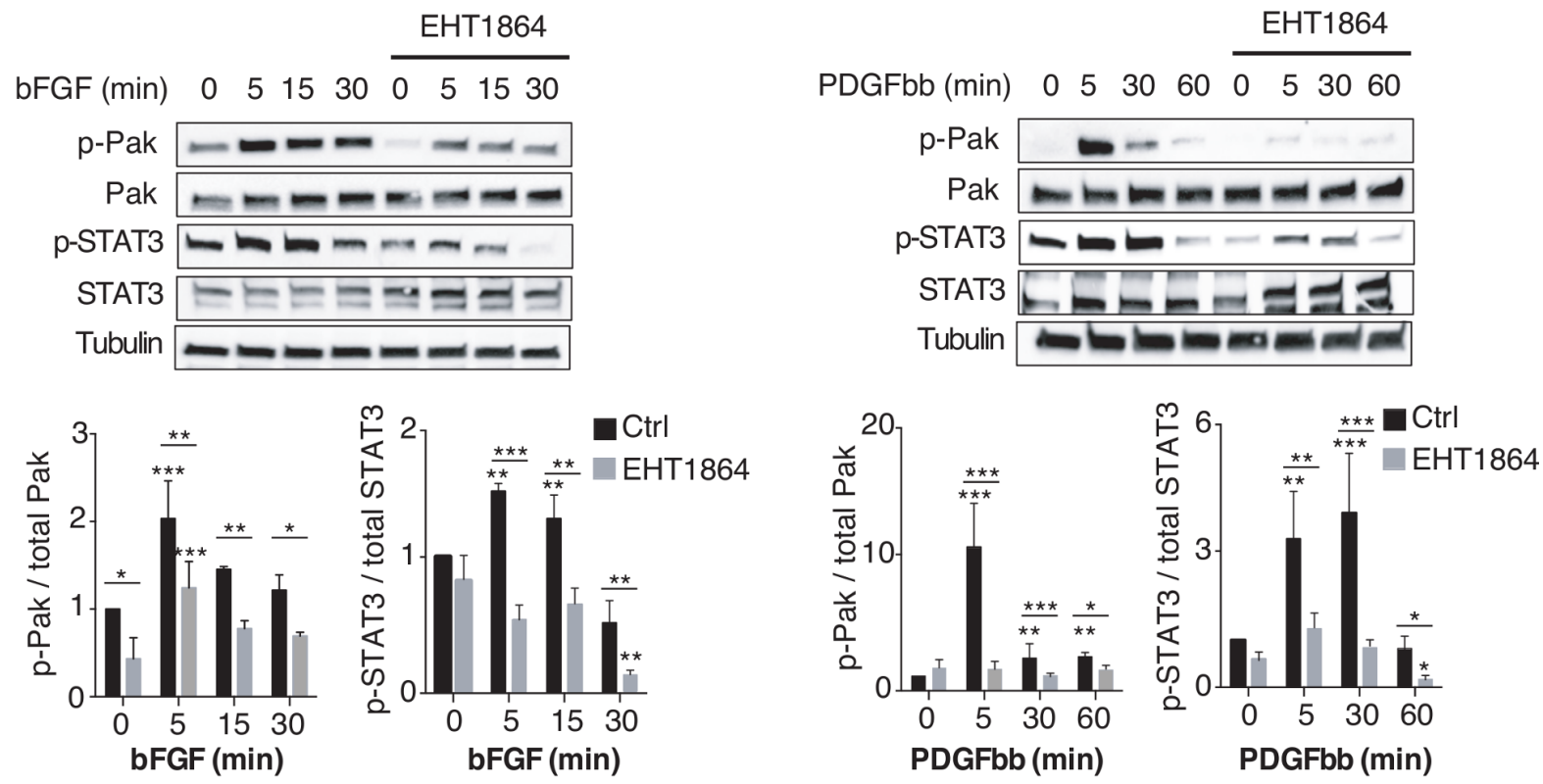

B
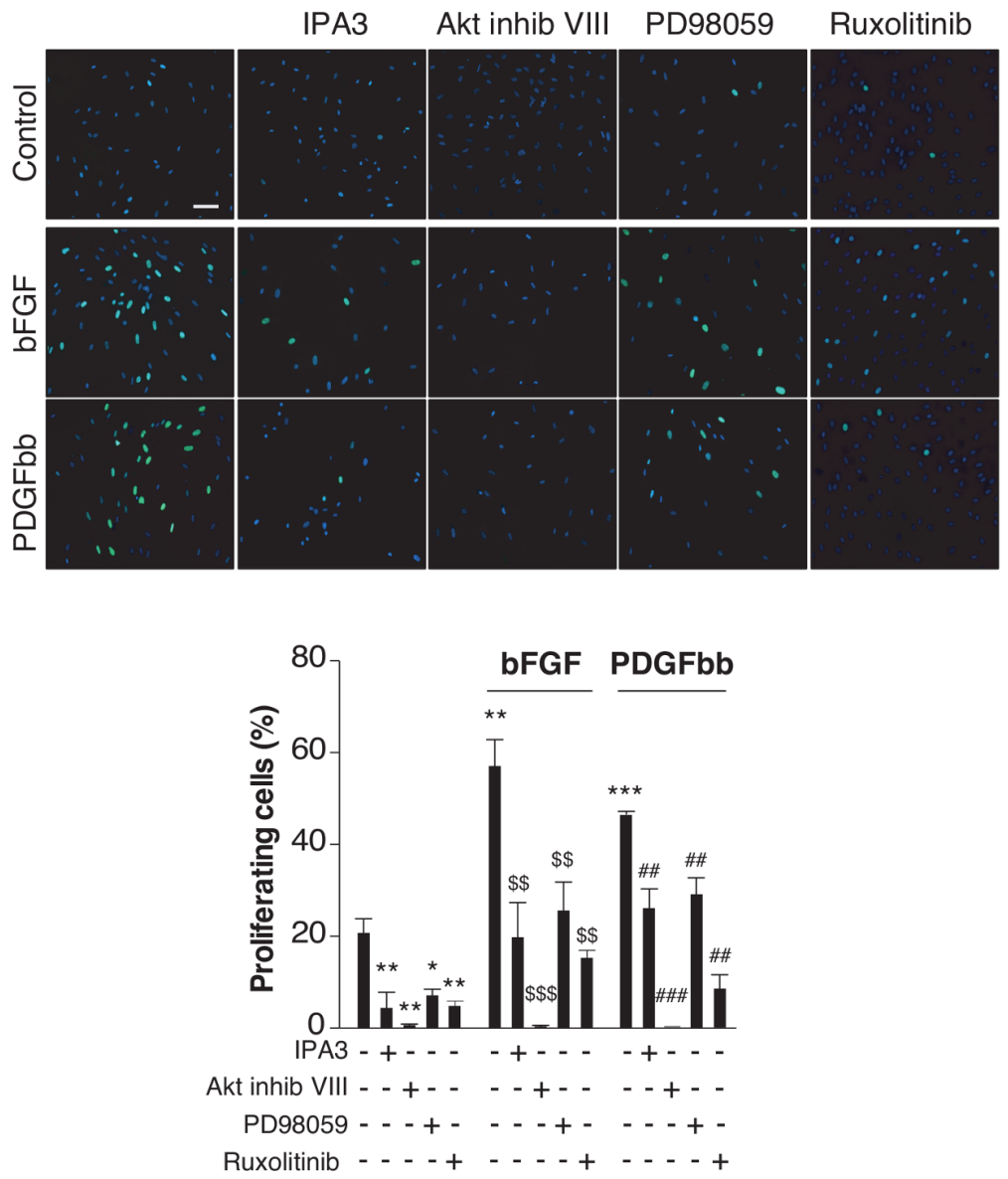

Figure 3 Role of Rac1/P21-activated kinases (Pak1) in bFGF-induced activation of Akt-dependent signalling pathway. (A) Immunoblot analysis and corresponding quantification of Pak and STAT3 expression and phosphorylation in control airway smooth muscle cell (aSMCs) stimulated with bFGF or PDGFbb at different time points, in the absence, or in the presence of EHT1864 ( $n=4-5$ independent experiments). (B) Control aSMC proliferation induced by bFGF and PDGFbb, in the absence and in the presence of inhibitors of PAK (IPA3), Akt (Akt Inhib VIII), P44/42 (PD98059) or JaAK2 (ruxolitinib). Nuclei are detected by 4',6-diamidino-2-phenylindole staining (blue) and haSMC proliferation by EdU staining (green). Scale bar, $25 \mu \mathrm{m}$. Quantification of aSMC proliferation by EdU staining. The results are expressed as the percentage of EdU-positive cells. ( $n=3-4$ independent experiments). Data are presented as mean \pm SEM. Kruskal-Wallis test followed by Dunns' post-test were used. ${ }^{*} \mathrm{P}<0.05,{ }^{* *} \mathrm{p}<0.01,{ }^{* * *} \mathrm{p}<0.001$ versus untreated cells; $; \$ p<0.01,{ }^{\$ \$ \$} p<0.001$ versus bFGF treated cells; ${ }^{* \#} p<0.01, " \#$ $p<0.001$ versus PDGFbb treated cells. 
A

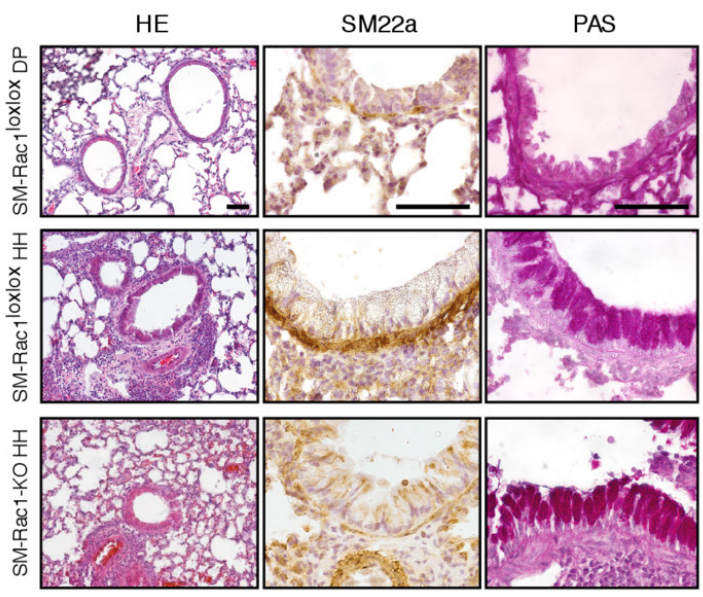

C

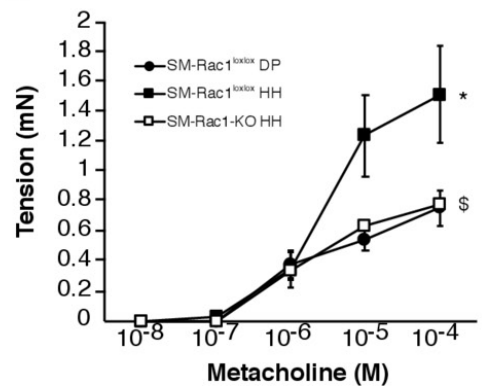

B
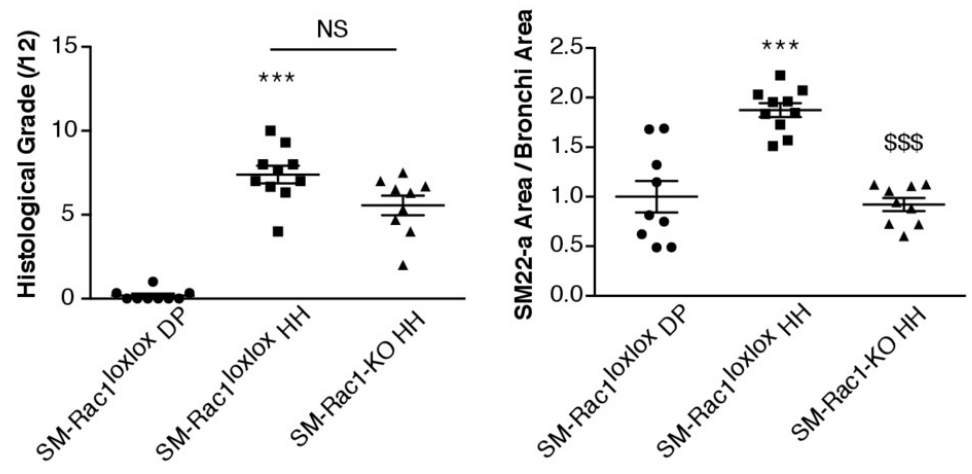

D

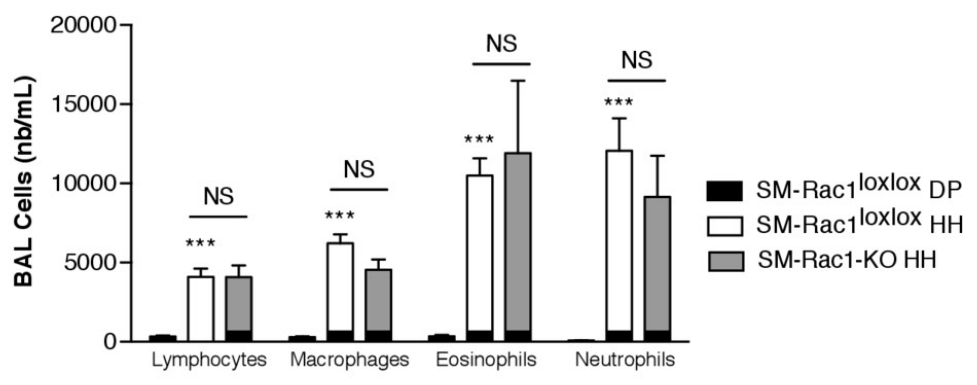

Figure 4 SM-Rac1 deletion prevents smooth muscle hyperplasia associated in an experimental model of severe allergic asthma. (A) Hematoxylin/ Eosin (HE staining, SM22a immunohistochemistry and Periodic acid-Schiff (PAS) staining of lung sections from naïve (DP) or house dust mitesensitised $(\mathrm{HH})$ mice of the indicated genotypes. Images are representative of 9-10 mice in each experimental condition. Scale bars, $100 \mu \mathrm{m}$. (B) Histological grade and smooth muscle hyperplasia quantification on lung sections from DP and HH mice of the indicated genotypes ( $\mathrm{n}=9-10$ mice). (C) Contractile responses to increasing concentration of methacholine of bronchial rings from DP and HH mice of the indicated genotypes ( $n=3-4)$. (D) Infiltrating cells in bronchoalveolar lavage fluid from DP and HH mice of the indicated genotypes $(n=8-14)$. Data are expressed as mean \pm SEM. Kruskal-Wallis test followed by Dunns' post-test were used for (B) and (D). Two-way analysis of variance test was used for (C). NS, not significant. ${ }^{*} \mathrm{P}<0.05,{ }^{* *} \mathrm{p}<0.001$ versus SM-Rac1 ${ }^{\text {lox/lox }}$ DP mice; ${ }^{\$} \mathrm{p}<0.05,{ }^{\$ \$ \$} \mathrm{p}<0.001$ versus SM-Rac1 ${ }^{\text {lox/lox }} \mathrm{HH}$ mice.

(benralizumab) and anti-IL-4/IL-13 receptor (dupilumab) significantly improve exacerbations rate and asthma control, and lower oral corticosteroid use in patients with severe asthma. ${ }^{37-41}$ However, these strategies focus on specific inflammatory endotypes, ${ }^{3942-44}$ and the potential impacts of these new strategies on airway remodelling are still missing. In this context, our demonstration that Rac1 is a node in signalling pathways that plays a major role in the contraction and proliferation of aSMC makes this protein a pharmacological target of choice for severe asthma. The efficiency of SMC Rac1 deletion and repeated inhalations of the Rac1 inhibitor NSC23766 to prevent AHR and aSMC hyperplasia validate this hypothesis in a severe allergic asthma model in mice that recapitulate the human disease. Moreover, in addition to these expected effects, Rac1 inhibition also reduced eosinophilic inflammation, thus demonstrating that the anti-inflammatory action of Rac1 inhibitor already described in an acute murine model of allergic asthma, ${ }^{14}$ is also effective in severe chronic asthma. To our knowledge, this is the first demonstration of a drug able to combine all the desired effects for the treatment of severe asthma, that is, limiting aSMC contraction and proliferation and reducing inflammation. Since Rac1 is known to have ubiquitous expression and multiple functions, ${ }^{45}$ an open and important question that remains to be addressed is the possible side effects of Rac1 inhibitor. However, in asthma, the opportunity of administrating the treatment locally might be an effective way to limit potential adverse effects. Indeed, repeated administration of
NSC23766 by nebulisation failed to alter blood pressure, whereas SMC Rac1 deletion has been shown to elicit this effect. ${ }^{46}$

In conclusion, we suggest that Rac1 may represent a relevant target to develop new drugs of clinical interest for the treatment of severe asthma. Our data support the concept that inhibition of Rac1-dependent signalling pathway may simultaneously limit aSMC hyperplasia, AHR and inflammation, thereby providing a novel approach to reverse airway remodelling and restore airway function in patients with severe asthma. The development of Rac1 inhibitors may thus offer a new therapeutic option for patients who are refractory to current treatments.

Acknowledgements The authors thank Marie-Aude Cheminant (institut du thorax) for expert technical assistance. We also value the support provided by the animal facility units of the University of Nantes. We thank Therassay, Micropicell and Cytocell core facilities (SFR François Bonamy, University of Nantes) for the functional and cellular explorations, Philippe LACOSTE (MD, PhD) and Megguy BERNARD (Nantes university hospital) for the collection of human bronchial samples from lung transplants. The authors also thank the support of the cluster LUNG innovation (LUNG 02; Programme d'Investissements d'Avenir ANR-16-IDEX-0007).

Contributors Conception and design : MP, AM, GL and VS. Experimentation: FD, $L R, D H, M K, M R, C B, C T, M C D, L D C$., NH, GB and VS. Analysis and interpretation: $F D, M P, G L$ and VS. Drafting the manuscript: FD, GL and VS. Authors FD and LR contributed equally to this work.

Funding This work was supported by grants from the Institut de Recherche en Santé Respiratoire des Pays de la Loire (G-Rar and NARACAS projects), the 
A

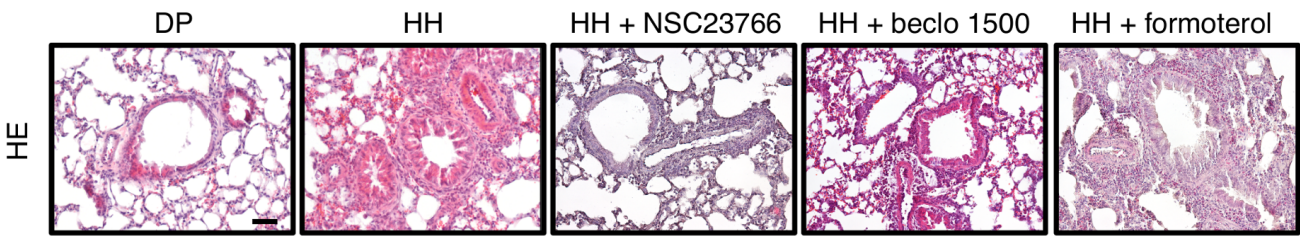

B
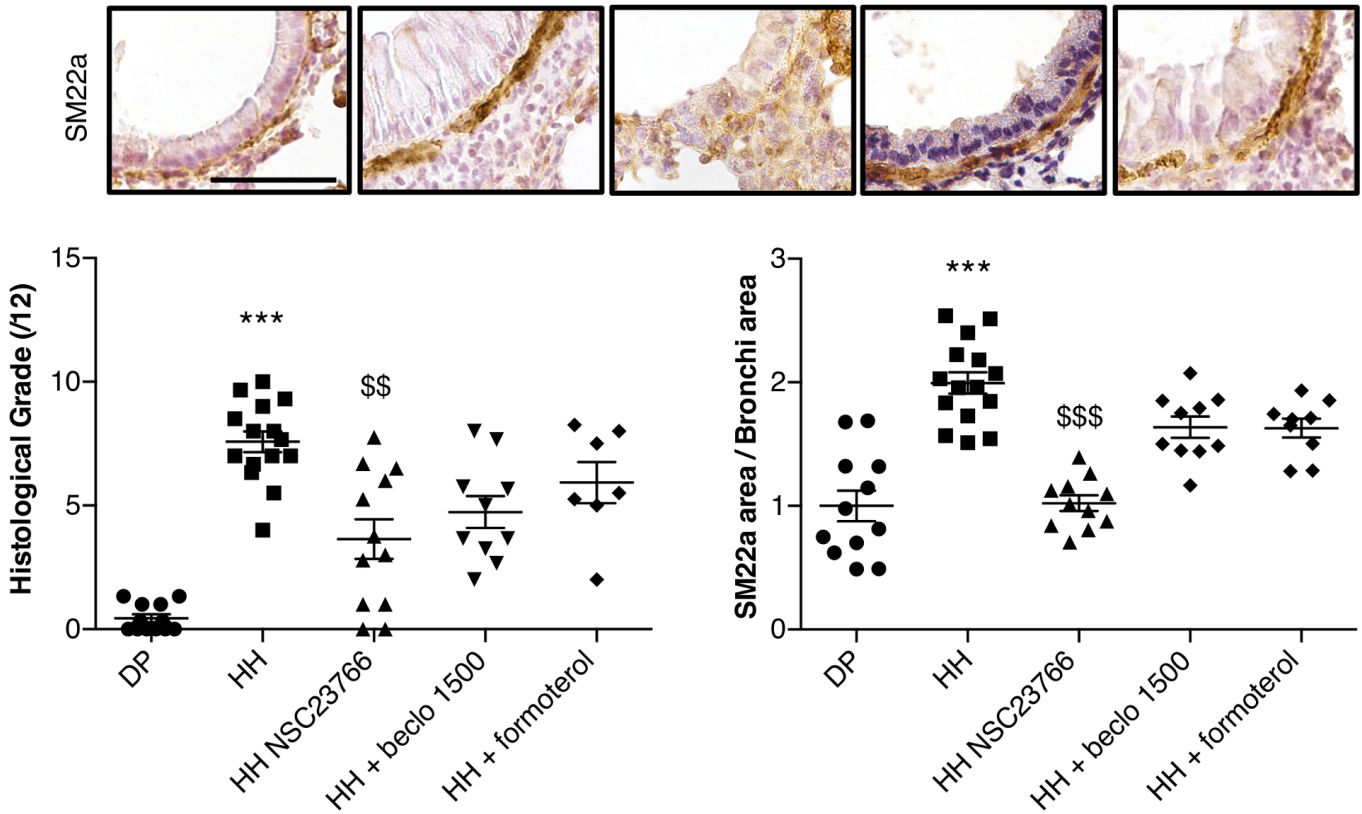

C
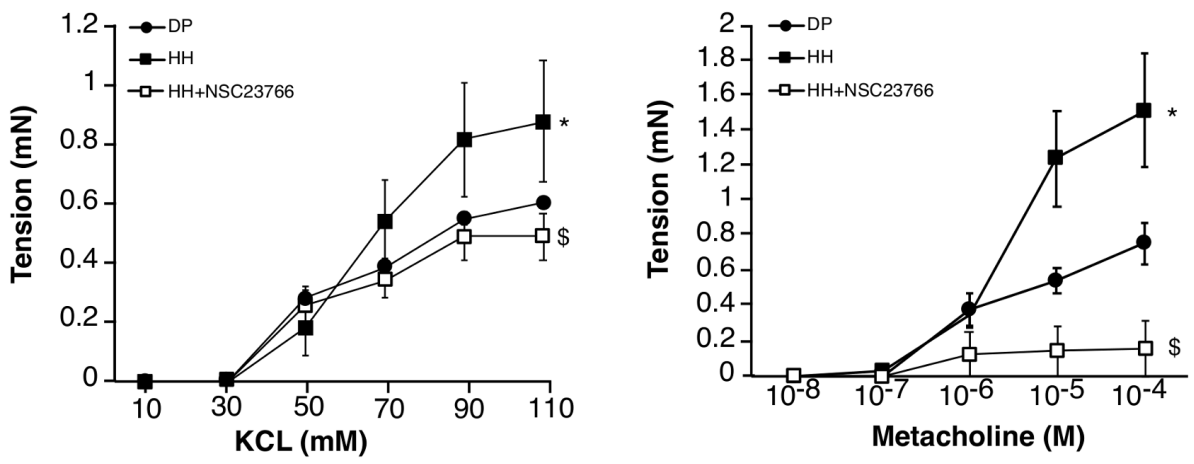

D

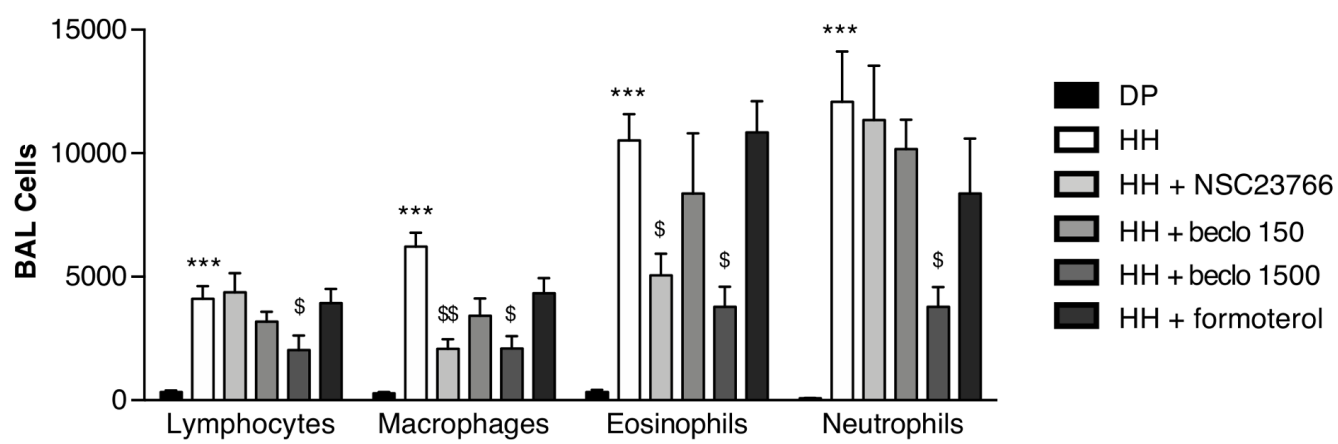

Figure 5 NSC23766 inhalations reduce pulmonary remodelling and smooth muscle hyperplasia associated with severe allergic asthma. (A) Hematoxylin/Eosin (HE) staining and SM22a immunohistochemistry of lung sections from naïve (DP) or house dust mite-sensitised (HH) mice treated with $\mathrm{NaCl}$, NSC23766, beclomethasone $(1500 \mu \mathrm{g} / \mathrm{kg})$ or formoterol. Images are representative of 9-15 mice in each experimental condition. Scale bars, $100 \mu \mathrm{m}$. (B) Histological grade and smooth muscle hyperplasia measured on lung sections from DP and HH mice treated with NaCl, NSC23766, beclomethasone or formoterol ( $\mathrm{n}=9-15$ mice). (C) Contractile responses to increasing concentrations of $\mathrm{KCl}$ and methacholine of bronchi rings from $\mathrm{DP}$ and $\mathrm{HH}$ mice treated with $\mathrm{NaCl}$ or NSC23766 $(\mathrm{n}=4-5)$. (D) Infiltrating cells in bronchoalveolar lavage fluid from DP and $\mathrm{HH}$ mice treated with $\mathrm{NaCl}$ NSC23766, beclomethasone (150 or $1500 \mu \mathrm{g} / \mathrm{kg}$ ), or formoterol ( $\mathrm{n}=9-14$ mice). Data are represented as mean \pm SEM. Kruskal-Wallis test followed by Dunns' post-test were used for (B) and (D). Two-way analysis of variance test was used for (c). ${ }^{*} \mathrm{P}<0.05,{ }^{* *} \mathrm{p}<0.001$ versus $\mathrm{DP} \mathrm{NaCl}$; ${ }^{\$} \mathrm{p}<0.05$, ${ }^{\$ \$} \mathrm{p}<0.01$ and ${ }^{\$ \$} \mathrm{p}<0.001$ versus $\mathrm{HH} \mathrm{NaCl}$. 
Société d'Accélération du Transfert de Technologie (project number STRAS-2117) and the Institut National de la Santé et de la Recherche Médicale (INSERM). LR was supported by a grant from MRES. FD and DH were supported by a grant from Fondation Recherche Médicale.

Competing interests None declared.

Patient consent for publication Not required.

Ethics approval All experimental procedures and animal care were performed in accordance with the Regional Ethical Committee for Animal Experiments of the Pays de la Loire and conform to the ARRIVE guidelines.

Provenance and peer review Not commissioned; externally peer reviewed.

Data availability statement All data relevant to the study are included in the article or uploaded as supplementary information. Vincent SAUZEAU, PhDvincent. sauzeau@inserm.fr.

Open access This is an open access article distributed in accordance with the Creative Commons Attribution Non Commercial (CC BY-NC 4.0) license, which permits others to distribute, remix, adapt, build upon this work non-commercially, and license their derivative works on different terms, provided the original work is properly cited, appropriate credit is given, any changes made indicated, and the use is non-commercial. See: http://creativecommons.org/licenses/by-nc/4.0/.

\section{REFERENCES}

1 Variations in the prevalence of respiratory symptoms, self-reported asthma attacks, and use of asthma medication in the European community respiratory health survey (ECRHS). Eur Respir J 1996;9:687-95.

2 Worldwide variation in prevalence of symptoms of asthma, allergic rhinoconjunctivitis, and atopic eczema: Isaac. The International study of asthma and allergies in childhood (Isaac) Steering Committee. Lancet Lond. Engl 1998;351:1225-32.

3 Reddel HK, Bateman ED, Becker A, et al. A summary of the new GINA strategy: a roadmap to asthma control. Eur Respir J 2015;46:622-39.

4 Chung KF, Wenzel SE, Brozek JL, et al. International ERS/ATS guidelines on definition, evaluation and treatment of severe asthma. Eur Respir J 2014;43:343-73.

5 Lane S, Molina J, Plusa T. An international observational prospective study to determine the cost of asthma exacerbations (COAX). Respir Med 2006;100:434-50.

6 Moore WC, Bleecker ER, Curran-Everett D, et al. Characterization of the severe asthma phenotype by the National heart, lung, and blood Institute's severe asthma research program. J Allergy Clin Immunol 2007;119:405-13.

7 Bento AM, Hershenson MB. Airway remodeling: potential contributions of subepithelial fibrosis and airway smooth muscle hypertrophy/hyperplasia to airway narrowing in asthma. Allergy Asthma Proc 1998;19:353-8.

8 Jeffery PK. Remodeling in asthma and chronic obstructive lung disease. Am J Respir Crit Care Med 2001;164:S28-38.

9 Prakash YS. Emerging concepts in smooth muscle contributions to airway structure and function: implications for health and disease. Am J Physiol Lung Cell Mol Physiol 2016;311:L1113-40

10 James AL, Bai TR, Mauad T, et al. Airway smooth muscle thickness in asthma is related to severity but not duration of asthma. Eur Respir J 2009;34:1040-5.

11 Castro M, Rubin A, Laviolette M, et al. Persistence of effectiveness of bronchial thermoplasty in patients with severe asthma. Ann Allergy Asthma Immunol 2011;107:65-70.

12 Castro M, Rubin AS, Laviolette M, et al. Effectiveness and safety of bronchial thermoplasty in the treatment of severe asthma: a multicenter, randomized, doubleblind, sham-controlled clinical trial. Am J Respir Crit Care Med 2010;181:116-24.

13 Thomson NC, Rubin AS, Niven RM, et al. Long-Term ( 5 year) safety of bronchial thermoplasty: asthma intervention research (air) trial. BMC Pulm Med 2011;11:8.

14 Liu Y, Li W, Ye C, et al. Gambogic acid induces G0/G1 cell cycle arrest and cell migration inhibition via suppressing PDGF receptor $\beta$ tyrosine phosphorylation and Rac1 activity in rat aortic smooth muscle cells. J Atheroscler Thromb 2010;17:901-13.

15 Page K, Li J, Hodge JA, et al. Characterization of a Rac, Signaling Pathway to Cyclin D ,Expression in Airway Smooth Muscle Cells. J Biol Chem 1999;274:22065-71.

16 Aubier M, Thabut G, Hamidi F, et al. Airway smooth muscle enlargement is associated with protease-activated receptor $2 /$ ligand overexpression in patients with difficult-tocontrol severe asthma. J Allergy Clin Immunol 2016;138:729-39.

17 André-Grégoire G, Dilasser F, Chesné J, et al. Targeting of Rac1 prevents bronchoconstriction and airway hyperresponsiveness. J Allergy Clin Immunol 2018;142:824-33.

18 Chesné J, Braza F, Chadeuf G, et al. Prime role of IL-17A in neutrophilia and airway smooth muscle contraction in a house dust mite-induced allergic asthma model. J Allergy Clin Immunol 2015;135:1643-5.

19 Hashimoto M, Tanaka H, Abe S. Quantitative analysis of bronchial wall vascularity in the medium and small airways of patients with asthma and COPD. Chest 2005:127:965-72
20 Hoshino M, Takahashi M, Aoike N. Expression of vascular endothelial growth factor, basic fibroblast growth factor, and angiogenin immunoreactivity in asthmatic airways and its relationship to angiogenesis. J Allergy Clin Immunol 2001;107:295-301.

21 Redington $A E$, Roche WR, Madden J, et al. Basic fibroblast growth factor in asthma: measurement in bronchoalveolar lavage fluid basally and following allergen challenge. J Allergy Clin Immunol 2001;107:384-7.

22 Vignola AM, Chanez P, Chiappara G, et al. Transforming growth factor-beta expression in mucosal biopsies in asthma and chronic bronchitis. Am J Respir Crit Care Med 1997; 156:591-9.

23 Bossé Y, Rola-Pleszczynski M. Fgf2 in asthmatic airway-smooth-muscle-cell hyperplasia. Trends Mol Med 2008;14:3-11.

24 Simeone-Penney MC, Severgnini M, Rozo L, et al. Pdgf-Induced human airway smooth muscle cell proliferation requires STAT3 and the small GTPase Rac1. Am J Physiol Lung Cell Mol Physiol 2008;294:L698-704.

25 Zhou L, Hershenson MB. Mitogenic signaling pathways in airway smooth muscle. Respir Physiol Neurobiol 2003;137:295-308.

26 Yap HM, Israf DA, Harith HH, et al. Crosstalk between signaling pathways involved in the regulation of airway smooth muscle cell hyperplasia. Front Pharmacol 2019;10:1148.

27 Jee S-H, Chu C-Y, Chiu H-C, et al. Interleukin-6 induced basic fibroblast growth factor-dependent angiogenesis in basal cell carcinoma cell line via JAK/STAT3 and PI3-kinase/Akt pathways. J Invest Dermato/ 2004;123:1169-75.

28 Shi $H$, Lin B, Huang $Y$, et al. Basic fibroblast growth factor promotes melanocyte migration via activating PI3K/Akt-Rac1-FAK-JNK and ERK signaling pathways. IUBMB Life 2016;68:735-47

29 Hrvacić B, Bosnjak B, Tudja M, et al. Applicability of an ultrasonic nebulization system for the airways delivery of beclomethasone dipropionate in a murine model of asthma. Pharm Res 2006;23:1765-75.

30 Johnson PR, Roth M, Tamm M, et al. Airway smooth muscle cell proliferation is increased in asthma. Am J Respir Crit Care Med 2001;164:474-7.

31 Bousoik E, Montazeri Aliabadi H. "Do We Know Jack" About JAK? A Closer Look at JAK/STAT Signaling Pathway. Front Oncol 2018;8:287.

32 Pelletier S, Duhamel F, Coulombe P, et al. Rho family GTPases are required for activation of JAK/STAT signaling by $\mathrm{G}$ protein-coupled receptors. Mol Cell Biol 2003;23:1316-33.

33 Movassagh H, Shan L, Halayko AJ, et al. Neuronal chemorepellent semaphorin 3E inhibits human airway smooth muscle cell proliferation and migration. J Allergy Clin Immunol 2014;133:560-7.

34 Huang $Y$, Xie $Y$, Jiang $H$, et al. Upregulated P-Rex 1 exacerbates human airway smooth muscle hyperplasia in asthma. J Allergy Clin Immunol 2019;143:778-81.

35 Buchman AL. Side effects of corticosteroid therapy. J Clin Gastroenterol 2001;33:289-94

36 Volmer T, Effenberger T, Trautner C, et al. Consequences of long-term ora corticosteroid therapy and its side-effects in severe asthma in adults: a focused review of the impact data in the literature. Eur Respir J 2018;52. doi:10.1183/13993003.00703-2018. [Epub ahead of print: 2510 2018].

37 Normansell R, Walker S, Milan SJ, et al. Omalizumab for asthma in adults and children. Cochrane Database Syst Rev 2014:CD003559.

38 Pavord ID, Korn S, Howarth P, et al. Mepolizumab for severe eosinophilic asthma (DREAM): a multicentre, double-blind, placebo-controlled trial. Lancet 2012;380:651-9.

39 Bleecker ER, FitzGerald JM, Chanez P, et al. Efficacy and safety of benralizumab for patients with severe asthma uncontrolled with high-dosage inhaled corticosteroids and long-acting $\beta 2$-agonists (SIROCCO): a randomised, multicentre, placebocontrolled phase 3 trial. The Lancet 2016;388:2115-27.

40 Castro M, Zangrilli J, Wechsler ME, et al. Reslizumab for inadequately controlled asthma with elevated blood eosinophil counts: results from two multicentre, parallel, double-blind, randomised, placebo-controlled, phase 3 trials. Lancet Respir Med 2015;3:355-66.

41 FitzGerald JM, Bleecker ER, Nair P, et al. Benralizumab, an anti-interleukin-5 receptor $\alpha$ monoclonal antibody, as add-on treatment for patients with severe, uncontrolled, eosinophilic asthma (CALIMA): a randomised, double-blind, placebo-controlled phase 3 trial. The Lancet 2016;388:2128-41.

42 FitzGerald JM, Bleecker ER, Menzies-Gow A, et al. Predictors of enhanced response with benralizumab for patients with severe asthma: pooled analysis of the SIROCCO and CALIMA studies. Lancet Respir Med 2018;6:51-64.

43 Haldar P, Brightling CE, Singapuri A, et al. Outcomes after cessation of mepolizumab therapy in severe eosinophilic asthma: a 12-month follow-up analysis. J Allergy Clin Immunol 2014;133:921-3.

44 Hanania NA, Wenzel S, Rosén K, et al. Exploring the effects of omalizumab in allergic asthma: an analysis of biomarkers in the extra study. Am J Respir Crit Care Med 2013;187:804-11.

45 Jaffe AB, Hall A. Rho GTPases: biochemistry and biology. Annu Rev Cell Dev Biol 2005;21:247-69.

46 André G, Sandoval JE, Retailleau K, et al. Smooth muscle specific Rac1 deficiency induces hypertension by preventing p116RIP3-dependent RhoA inhibition. J Am Heart Assoc 2014;3:e000852. 